\title{
Pseudo-transient 3D Conjugate Heat Transfer Simulation and Lifetime Prediction of a Rocket Combustion Chamber
}

\author{
Oliver Barfusz, Felix Hötte, Stefanie Reese, and Matthias Haupt
}

\begin{abstract}
Rocket engine nozzle structures typically fail after a few engine cycles due to the extreme thermomechanical loading near the nozzle throat. In order to obtain an accurate lifetime prediction and to increase the lifetime, a detailed understanding of the thermomechanical behavior and the acting loads is indispensable. The first part is devoted to a thermally coupled simulation (conjugate heat transfer) of a fatigue experiment. The simulation contains a thermal FEM model of the fatigue specimen structure, RANS simulations of nine cooling channel flows and a Flamelet-based RANS simulation of the hot gas flow. A pseudo-transient, implicit Dirichlet-Neumann scheme is utilized for the partitioned coupling. A comparison with the experiment shows a good agreement between the nodal temperatures and their corresponding thermocouple measurements. The second part consists of the lifetime prediction of the fatigue experiment utilizing a sequentially coupled thermomechanical analysis scheme. First, a transient thermal analysis is carried out to obtain the temperature field within the fatigue specimen. Afterwards, the computed temperature serves as input for a series of quasi-static mechanical analyses, in which a viscoplastic damage model is utilized. The evolution and progression of the damage variable within the regions of interest are thoroughly discussed. A comparison between simulation and experiment shows that the results are in good agreement. The crucial failure mode (doghouse effect) is captured very well.
\end{abstract}

\footnotetext{
O. Barfusz (凶) · S. Reese

RWTH Aachen University, Institute of Applied Mechanics,

Mies-van-der-Rohe-Straße 1, 52074 Aachen, Germany

e-mail: oliver.barfusz@rwth-aachen.de

\section{S. Reese}

e-mail: stefanie.reese@rwth-aachen.de

F. Hötte $\cdot$ M. Haupt

TU Braunschweig, Institute of Aircraft Design and Lightweight Structures, Hermann-Blenk-Str. 35, 38108 Braunschweig, Germany

e-mail: f.hoette@tu-braunschweig.de

M. Haupt

e-mail:m.haupt@tu-braunschweig.de
} 


\section{Introduction}

The Transregio 40 subproject D9's fatigue experiment (see [13, 14]) is used as reference configuration. It serves as a basis for extensive measurements which are further used for validation. In this fatigue experiment a replaceable fatigue specimen made of $\mathrm{CuCr} 1 \mathrm{Zr}$ is mounted downstream of a rectangular GOX/GCH4 combustion chamber (see Fig. 1a). The fatigue specimen is cooled by mass flow and pressure regulated supercritical nitrogen in its 17 cooling channels. The load is cyclical and consists of pre-cooling, hot-run and post-cooling phase. The hot-run phase is further divided into two pressure stages. After each cycle the deformations of the hot gas exposed surface are measured by a laser-profile-scanner. The fatigue specimen is equipped with several thermocouples. Figure $1 \mathrm{~b}$ shows the position of these thermocouples T6-T23 in the top view of the fatigue specimen. The distance to the hot gas exposed surface is $3 \mathrm{~mm}$ for the red marked and $5 \mathrm{~mm}$ for the blue marked thermocouples.

For lifetime predictions of rocket combustion chamber structures the knowledge of the transient temperature field of the structure is required. The most common method is to replace the coolant and hot gas flow by heat transfer coefficients in a thermal structural model. For estimating the heat transfer coefficients the use of Nusselt number correlations is strongly limited to simple configurations. For other configurations the heat transfer coefficients have to be derived from temperature

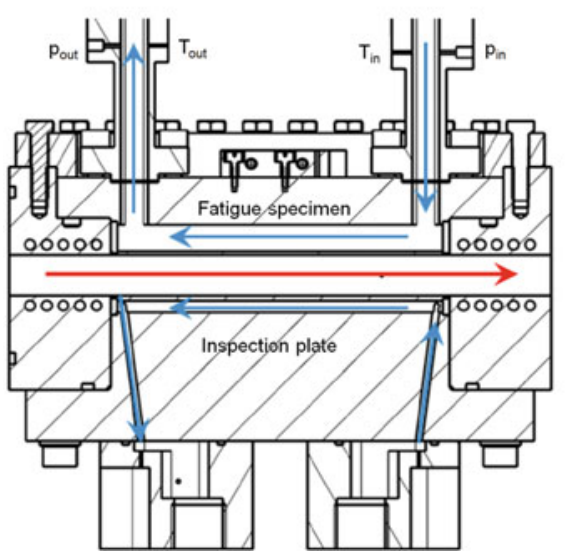

(a) Fatigue segment cut view (Reprinted by permission from Springer Nature [10]), cut position marked as A-A in Fig. 1(b)

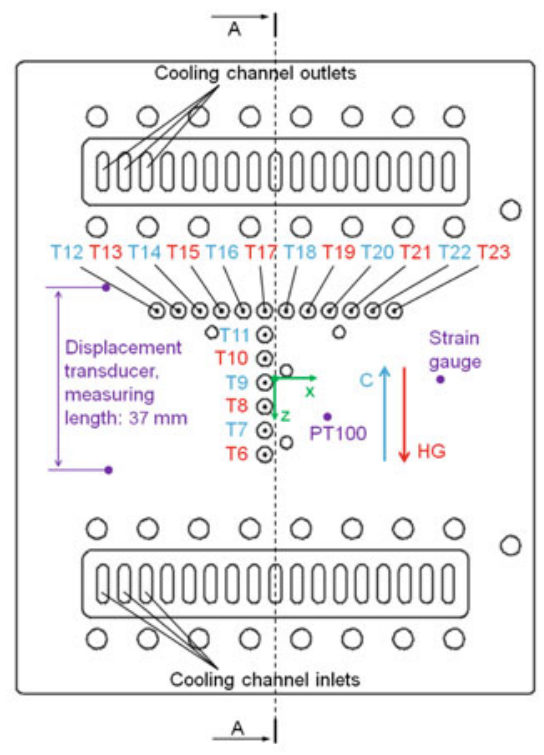

(b) Fatigue specimen top view with flow directions and measurement positions (Reprinted by permission from Felix Hötte [13])

Fig. 1 Set-up of the fatigue experiment 
measurements. In this paper a 3D Conjugate Heat Transfer (CHT) simulation is presented, which is able to predict the transient temperature fields of complex configurations without the need of experimental data fitting. Besides its use for lifetime predictions, the simulation can increase the understanding and close the gaps of the experimental lifetime investigations.

\section{Conjugate Heat Transfer Simulation}

\subsection{Computational Model}

Due to symmetry, the CHT model includes half of the fatigue specimen, eight and a half cooling channel flows and a quarter of the hot gas flow. For the coolant flow simulation the Finite Volume Method (FVM) based open source Computational Fluid Dynamics (CFD) tool OpenFOAM is utilized. On structural side the Finite Element Method (FEM) based commercial Computational Solid Mechanics (CSM) tool Abaqus is used. For the hot gas flow simulation the FVM based commercial CFD tool Ansys Fluent is applied. For controlling the field solvers and managing the data exchange between the 11 domains the in-house tool ifls [18] together with the Dirichlet-Neumann coupling scheme is utilized. On the coupling surface heat fluxes, calculated in the fluid domain boundaries, are applied as boundary condition for the solid domain. Vice versa, temperatures, calculated in the solid domain boundaries, are utilized as boundary condition for the fluid domains. The coupling is implicit, therefore the interface quantities will be exchanged during one time step until convergence. Aitken's dynamic relaxation is applied to accelerate the convergence of the equilibrium iteration. It is assumed that the time scales of the fluid domains are much smaller than the time scales of the structure domain. Therefore, the structure domain is solved transiently, whereas the fluid domains are solved under the assumption of steady-state conditions. The reader is referred to [12] for a detailed description of the governing equations and the material models used for the different domains.

For the simulation of the coolant domain the OpenFOAM steady-state solver buoyantSimpleFoam with the BSL-EARSM turbulence model [17] is utilized. The material data are considered to be temperature dependent. The turbulent Prandtl number is assumed as 0.85 . Figure 2 a shows the computational domain of the half of the central cooling channel. Due to simplicity the original cross section of the inlet and outlet channel was modified from an elliptical to a rectangular cross section without changing the hydraulic diameter. Table 1 shows the boundary conditions. $n$ indicates the normal vector of the surfaces. $U_{n}$ and $U_{p}$ are the velocity components in direction $n$ and in plane direction. $T$ is the temperature, $p$ the pressure, $\omega$ the turbulence specific dissipation, $k$ the turbulence kinetic energy, $v_{t}$ the turbulent kinematic viscosity, $\alpha_{t}$ the turbulent thermal diffusivity, $v$ the kinematic viscosity and $y$ the wall distance of the cell center. The temperature of the walls is calculated in the structure domain and substituted iteratively. All other cooling channel domains are consisting of this half 


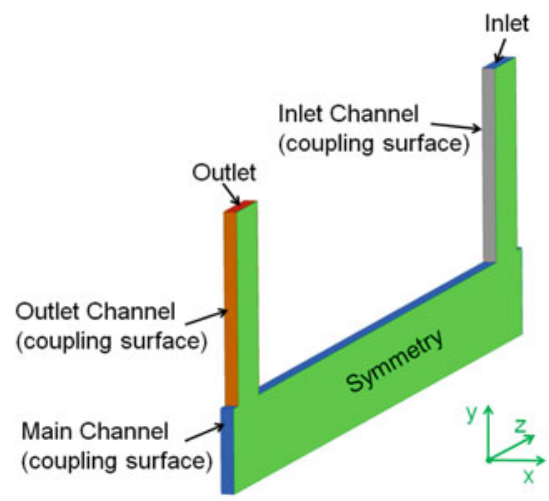

(a) Coolant domain

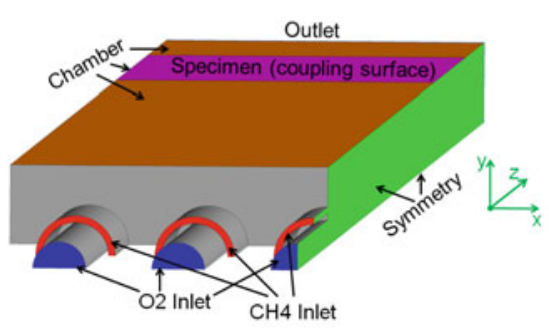

(b) Hot gas domain

Fig. 2 Boundary conditions of the fluid domains

Table 1 Boundary conditions for the coolant flow simulation

\begin{tabular}{l|l|l|l|l}
\hline Variable & Inlet & Outlet & $\begin{array}{l}\text { Walls (coupling } \\
\text { surfaces) }\end{array}$ & Symmetry \\
\hline$T, \mathrm{~K}$ & 286.5 & $\frac{\partial T}{\partial n}=0$ & $T_{\text {Struct }}$ & $\frac{\partial T}{\partial n}=0$ \\
\hline$U_{n}, \mathrm{~m} / \mathrm{s}$ & 5.6175 & $\frac{\partial U_{n}}{\partial n}=0$ & 0 & 0 \\
\hline$U_{p}, \mathrm{~m} / \mathrm{s}$ & 0 & $\frac{\partial U_{p}}{\partial n}=0$ & 0 & $\frac{\partial U_{p}}{\partial n}=0$ \\
\hline$p, b a r$ & $\frac{\partial p}{\partial n}=0$ & 70 & $\frac{\partial p}{\partial n}=0$ & $\frac{\partial p}{\partial n}=0$ \\
\hline$\omega, 1 / \mathrm{s}$ & 2465 & $\frac{\partial \omega}{\partial n}=0$ & $\frac{80 v}{y^{2}}$ & $\frac{\partial \omega}{\partial n}=0$ \\
\hline$k, \mathrm{~m}^{2} / \mathrm{s}^{2}$ & 0.1183 & $\frac{\partial k}{\partial n}=0$ & $10^{-10}$ & $\frac{\partial k}{\partial n}=0$ \\
\hline$v_{t}, \mathrm{~m}^{2} / \mathrm{s}$ & $/$ & $/$ & 0 & $\frac{\partial v_{t}}{\partial n}=0$ \\
\hline$\alpha_{t}, \mathrm{~kg} /(\mathrm{m} \cdot \mathrm{s})$ & 0 & $\frac{\partial \alpha_{t}}{\partial n}=0$ & 0 & $\frac{\partial \alpha_{t}}{\partial n}=0$ \\
\hline & & & & \\
\hline
\end{tabular}

cooling channel model and its counterpart mirrored with respect to the symmetry plane. A computational grid with 500,296 block-structured cells and a dimensionless wall distance $y^{+}$of in average 0.32 is used for the central cooling channel. Each grid of the eight other cooling channels consists of 1,000,592 block-structured cells per channel.

For simulating the hot gas domain Fluent's steady state pressure-based solver is utilized. For modeling the Reynolds stresses the standard $k-\epsilon$-model by Jones and Launder [15] with a two-layer approach in the near-wall region (see [1]) is applied. A non-adiabatic steady diffusion Flamelet model is used in a preprocessing step to calculate the relationships of the instantaneous temperature, density and species mass fractions to the total enthalpy, mixture fraction and its variance. The kinetic chemistry scheme of [21] is applied, containing 21 species and 97 reactions. A $\beta$-probability density function is assumed, to describe the turbulent, temporal fluctuations of the mixture fraction. Figure $2 \mathrm{~b}$ shows the hot gas domain and Table 2 its boundary 
Table 2 Boundary conditions for the hot gas flow simulation

\begin{tabular}{l|l|l|l|l|l|l|l}
\hline Variable & O2 Inlet & CH4 Inlet & Outlet & Chamber & Specimen & Symmetry & Others \\
\hline$T, \mathrm{~K}$ & 275.6 & 261.2 & $\frac{\partial T}{\partial n}=0$ & 500 & $T_{\text {Struct }}$ & $\frac{\partial T}{\partial n}=0$ & $\frac{\partial T}{\partial n}=0$ \\
\hline$U_{n}, \mathrm{~m} / \mathrm{s}$ & $57.6,129.5$ & $41.0,93.9$ & $\frac{\partial U_{n}}{\partial n}=0$ & 0 & 0 & 0 & 0 \\
\hline$U_{p}, \mathrm{~m} / \mathrm{s}$ & 0 & 0 & $\frac{\partial U_{p}}{\partial n}=0$ & 0 & 0 & $\frac{\partial U_{p}}{\partial n}=0$ & 0 \\
\hline$p, b a r$ & $\frac{\partial p}{\partial n}=0$ & $\frac{\partial p}{\partial n}=0$ & $7.31,18.9$ & $\frac{\partial p}{\partial n}=0$ & $\frac{\partial p}{\partial n}=0$ & $\frac{\partial p}{\partial n}=0$ & $\frac{\partial p}{\partial n}=0$ \\
\hline$k, \mathrm{~m}^{2} / \mathrm{s}^{2}$ & $12.4,62.9$ & $6.3,33.1$ & $\frac{\partial k}{\partial n}=0$ & $\mathrm{WF}$ & $\mathrm{WF}$ & $\frac{\partial k}{\partial n}=0$ & $\mathrm{WF}$ \\
\hline$\epsilon, 10^{5} \mathrm{~m}^{2} / \mathrm{s}^{3}$ & $0.26,2.93$ & $0.37,4.46$ & $\frac{\partial \epsilon}{\partial n}=0$ & $\mathrm{WF}$ & $\mathrm{WF}$ & $\frac{\partial \epsilon}{\partial n}=0$ & $\mathrm{WF}$ \\
\hline $\bar{f},-$ & 0 & 1 & $\frac{\partial \bar{f}}{\partial n}=0$ & $\frac{\partial \bar{f}}{\partial n}=0$ & $\frac{\partial \bar{f}}{\partial n}=0$ & $\frac{\partial \bar{f}}{\partial n}=0$ & $\frac{\partial \bar{f}}{\partial n}=0$ \\
\hline$\overline{f^{\prime 2}},-$ & 0 & 0 & $\frac{\partial f^{\prime 2}}{\partial n}=0$ & 0 & 0 & $\frac{\partial f^{\prime 2}}{\partial n}=0$ & 0 \\
\hline
\end{tabular}

In case of two entries per column: first entry $\hat{=}$ ignition stage, second entry $\hat{=}$ main stage

conditions for both pressure stages. $\epsilon$ is the rate of dissipation of turbulence energy, $\bar{f}$ is the Favre mean mixture fraction and $\overline{f^{\prime 2}}$ its variance. The temperature of the specimen is calculated in the structure domain and substituted iteratively. In Table 2 WF means that the turbulence quantities at the walls are calculated by the Ansys Fluent Advanced Wall Treatment (see [1] for details). A computational grid with $1.39 \cdot 10^{6}$ block-structured cells and a dimensionless wall distance $y^{+}$of in average 0.83 is used.

Abaqus Standard is applied to solve the transient heat conduction inside the structure. The temperature dependence of the material data for $\mathrm{CuCr} 1 \mathrm{Zr}$ is considered. Figure 3 shows the boundary conditions of the structural domain. The coupling surface to the hot gas and coolant are colored red and blue. All other surfaces, including the green symmetry plane are assumed as adiabatic. Equivalent to the coolant simulation, but inconsistent with the experiment, the inlet and outlet channel's cross sections are rectangular. In addition, the notches and the holes for the screws and thermocouples are neglected. The mesh consists of 372,670 nodes and 322,266 linear brick elements.

\subsection{Results and Validation}

Figure 4a shows the temperature transients for thermocouple T6 (see Fig. 1b) and its equivalent nodes for two simulation cases. After ignition at $\mathrm{t}=0 \mathrm{~s}$ the temperature increases with a decreasing gradient. At $t=7.5 \mathrm{~s}$ the second load stage starts, which increases the temperature gradient rapidly. After that the gradient decreases again until $\mathrm{t}=27.5 \mathrm{~s}$, where the flames are extinguished and the maximum temperatures are reached. The temperatures decrease rapidly after extinguishing.

In both simulation cases the turbulent Prandtl number $P r_{t}$ of the hot gas domain is varied between 0.7 and 0.9. A lower $P r_{t}$ increases the wall heat flux. For $P r_{t}=0.7$ the transient behaviour agrees very well with the experiment. The maximum deviation 


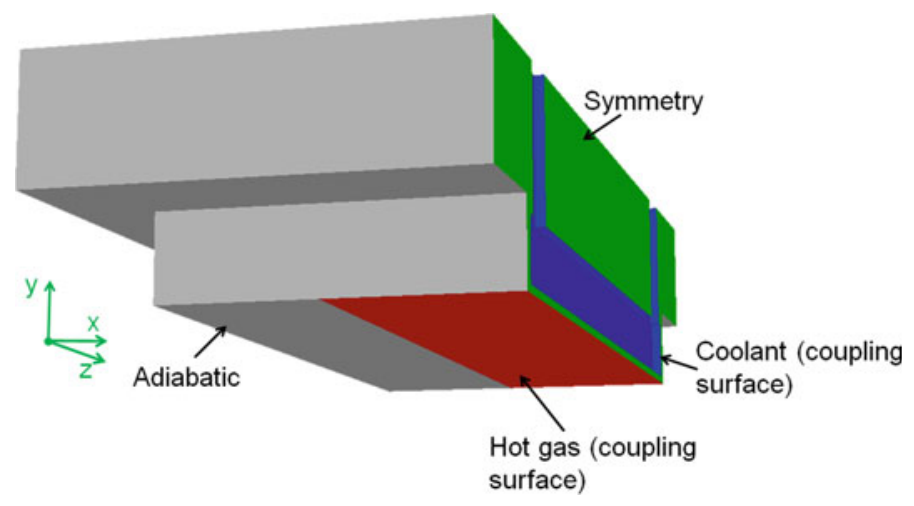

Fig. 3 Boundary conditions of the structure domain

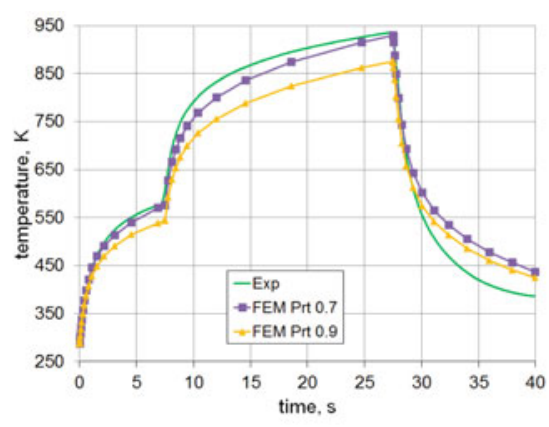

(a) Temperature transients for thermocouple T6 and its equivalent node

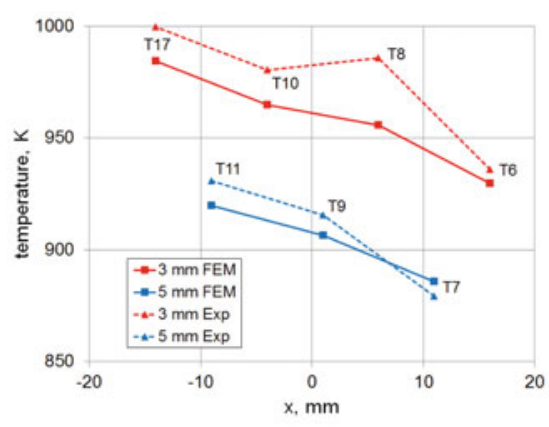

(b) Axial temperature distribution $\left(P r_{t}=0.7\right)$

Fig. 4 Comparison of thermocouple measurements and its corresponding FEM node temperatures

of $34 \mathrm{~K}$ during the hot run is reached after $10.4 \mathrm{~s}$. After that the deviation is decreasing. During the post-cooling phase the deviation is increasing again up to $69 \mathrm{~K}$. Therefore, it can be concluded, that the heat fluxes of the coolant and hot gas domain are slightly underestimated.

Figure 4a shows the axial temperature distribution at the end of the hot gas run $(\mathrm{t}=27.5 \mathrm{~s})$ for experiment and simulation $\left(P r_{t}=0.7\right)$. The red curves show the temperature distribution in $3 \mathrm{~mm}$ distance to the hot gas exposed surface and the blue curves in $5 \mathrm{~mm}$ distance, respectively. The temperature is decreasing in $\mathrm{x}$-direction (in hot gas flow direction). This is mostly caused by the high heat flux of the bumping coolant in the cooling channel inlet region (counterflow cooling).

The temperature distribution shows a good agreement between simulation and experiment. The vertical temperature difference between 3 and $5 \mathrm{~mm}$ distance to the hot gas exposed surface is around $50 \mathrm{~K}$ in the experiment and around $45 \mathrm{~K}$ in the simulation. This also indicates that the heat flux is slightly underestimated in the fluid domains. The axial temperature gradient agrees well. 


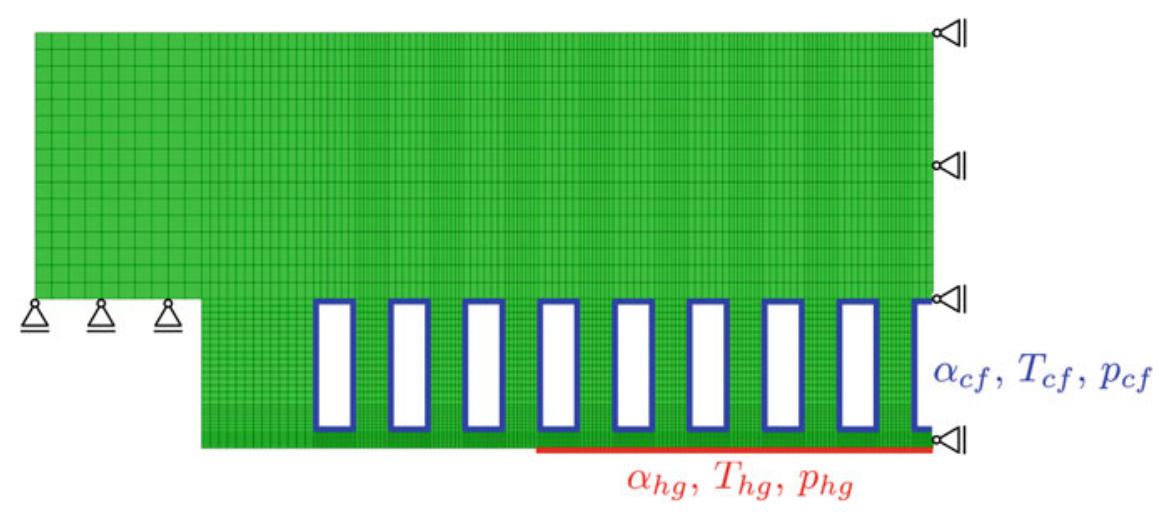

Fig. 5 FE model of the fatigue specimen with thermal and mechanical boundary conditions

\section{Lifetime Prediction}

For the lifetime prediction of the fatigue experiment a sequentially coupled analysis scheme is used which means that the analysis is split into two parts. First, a transient thermal analysis is carried out (see Sect.3.1) to obtain the temperature field within the specimen in every time step. The computed temperature then serves as input for a series of quasi-static mechanical analyses (see Sect.3.2), in which a viscoplastic damage model (see e.g. Kowollik et al. [16] and Fassin et al. [10]) is utilized. The Finite Element (FE) model with corresponding boundary conditions used for the thermal and mechanical analyses is illustrated in Fig. 5.

\subsection{Transient Thermal Analysis}

The thermal boundary conditions at the cooling channels and the hot gas wall for one cycle with a total duration of $60 \mathrm{~s}$ are summarized in Table 3. Therein, $\alpha_{h g}, \alpha_{c f}, T_{h g}$, and $T_{c f}$ denote the convective heat transfer coefficients and the bulk temperatures of the hot gas $(h g)$ side and the cooling fluid $(c f)$, respectively. Figure 6 shows the temperature distribution within the specimen reaching a maximum of $1113.18 \mathrm{~K}$ at the hot gas wall after the second hot run phase (i.e. 29.5 s). Furthermore, the temperature evolution over time at three different positions which are close to the region of interest where failure is expected to occur is illustrated and compared to experimental results coming from the thermocouple measurements T17, T18 and T20 (cf. Fig. 1). It can be observed that the numerical and experimental results agree very well, especially close to the symmetry plane (i.e. T17 and T18). For T20 a slight deviation can be recognized where higher temperatures are reached in the simulation, especially after the two hot run phases. This stems from the fact that due to simplicity and the lack of experimental data, the hot gas film coefficient $\alpha_{\mathrm{hg}}$ was 
Table 3 Thermal boundary conditions for one cycle

\begin{tabular}{l|l|l|l|l|l}
\hline Phase $(-)$ & Time $(\mathrm{s})$ & $\alpha_{\mathrm{hg}}\left(\frac{\mathrm{mW}}{\left(\mathrm{K} \mathrm{mm}^{2}\right)}\right)$ & $T_{\mathrm{hg}}(\mathrm{K})$ & $\alpha_{\mathrm{cf}}\left(\frac{\mathrm{mW}}{\left(\mathrm{K} \mathrm{mm}^{2}\right)}\right)$ & $T_{\mathrm{cf}}(\mathrm{K})$ \\
\hline Pre cooling & $0.0-2.0$ & - & 293 & 1.3208 & 288 \\
\hline Hot run 1 & $2.0-9.5$ & 2.543 & 2400 & 1.3208 & 288 \\
\hline Hot run 2 & $9.5-29.5$ & 5.066 & 2400 & 1.3208 & 288 \\
\hline Post cooling & $29.5-60.0$ & $3.0-10.0$ & 250 & 1.3208 & 288 \\
\hline
\end{tabular}

chosen to be constant during each hot run phase (cf. Table 3). This, however, is not of major concern, since final failure is expected to occur close to the symmetry plane which will be further investigated in Sect.3.2.

\subsection{Quasi-static Mechanical Analysis}

The lifetime of the fatigue specimen which is made of a copper alloy (CuCr1Zr) is limited by the failure of the cooling channels. Therefore the material and damage modeling of $\mathrm{CuCr} 1 \mathrm{Zr}$ is of major concern. To this end, a viscoplastic damage model presented in former works (see e.g. Kowollik et al. [16] or Fassin et al. [10]) is utilized which accounts for nonlinear kinematic and isotropic hardening, respectively. Furthermore, rate-dependence of Perzyna type and Lemaitre type ductile damage are incorporated into the model. The applied material parameters are chosen as in Fassin et al. [9] and Barfusz et al. [3]. Using a staggered simulation scheme, the thermomechanical coupling is performed only in one way considering the influence of the temperature on the mechanical behavior, but not vice versa. Temperature dependence of the mechanical analysis is taken into account by thermal expansion and the temperature dependence of the material parameters. The temperature field resulting from the thermal analysis (see Sect. 3.1) serves as an input for the quasistatic simulation consisting of multiple cycles.

The mechanical boundary conditions at the cooling channels and the hot gas wall are given in Table 4. It contains the respective pressures $p_{h g}$ and $p_{c f}$. Efficient and robust low-order continuum finite elements based on reduced integration with hourglass stabilization ${ }^{1}$ are used for the spatial discretization. Due to computational efficiency, only one element is utilized in depth direction whereby the displacement in this direction is fixed, leading to a plane strain state. Since the simulation is aborted at the beginning of the 48th cycle (no convergence), the following results are only shown up to the 47 th cycle.

Figure 7 illustrates the contour of the scalar damage variable $D$ after 47 cycles, ranging from the undamaged state $(D=0)$ to the fully damaged state $(D=1)$, the latter of which corresponds to macroscopic failure. It can be observed that the wall

\footnotetext{
${ }^{1} \mathrm{C} 3 \mathrm{D} 8 \mathrm{R}$ formulation within the commercial solver Abaqus.
} 

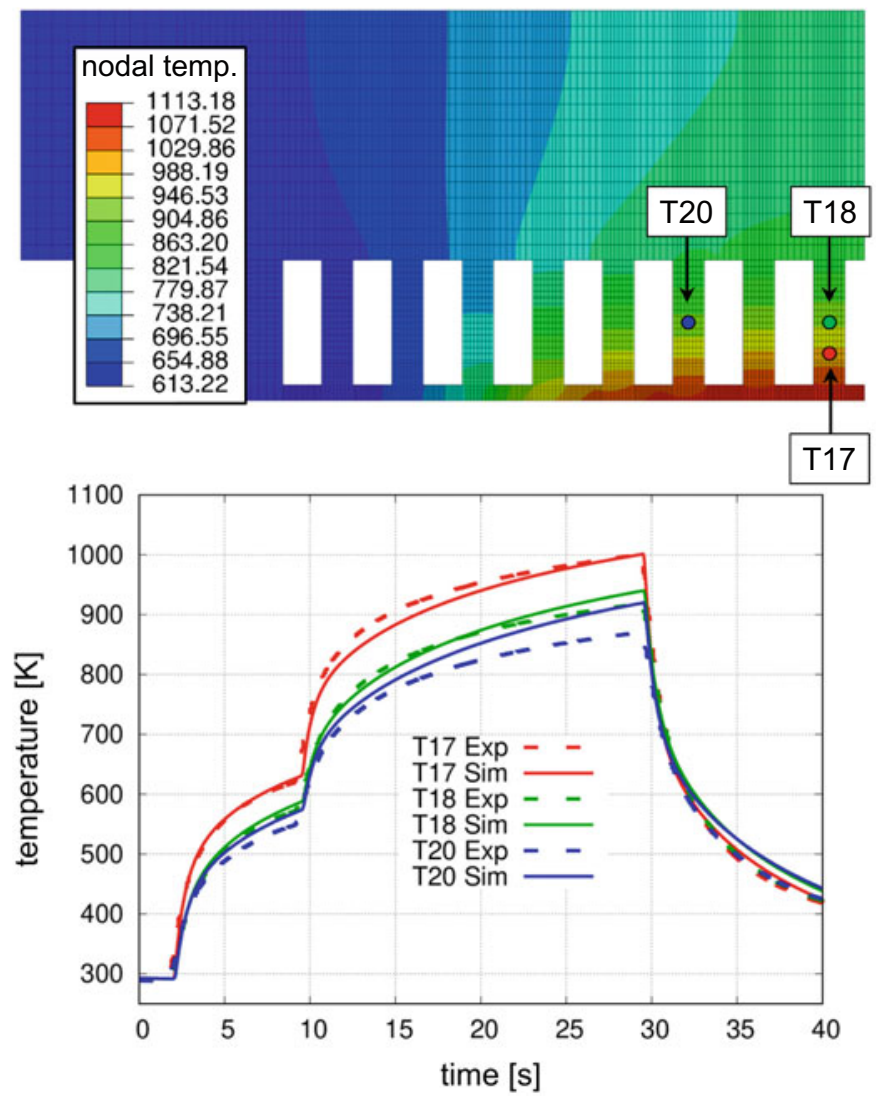

Fig. 6 Thermal analysis results-(top) snapshot of the temperature distribution after $29.5 \mathrm{~s}$ and positions of considered thermocouples (cf. Fig. 1); (bottom) evolution of the temperature during one cycle at considered thermocouples and validation with experiment

Table 4 Mechanical boundary conditions for one cycle

\begin{tabular}{l|l|l|l}
\hline Phase $(-)$ & Time $(\mathrm{s})$ & $p_{\text {hg }}(\mathrm{MPa})$ & $p_{\text {cf }}(\mathrm{MPa})$ \\
\hline Pre cooling & $0.0-2.0$ & - & 7.0 \\
\hline Hot run 1 & $2.0-9.5$ & 0.9 & 7.0 \\
\hline Hot run 2 & $9.5-29.5$ & 1.7 & 7.0 \\
\hline Post cooling & $29.5-60.0$ & - & 7.0 \\
\hline
\end{tabular}

between the first two cooling channels next to the symmetry plane and the hot gas side represents the location of maximum damage accumulation (i.e. material degradation). Zooming into the region of interest after several cycles, the degradation process can be well monitored. Starting at the cooling side edge (cycle 42), damage grows 


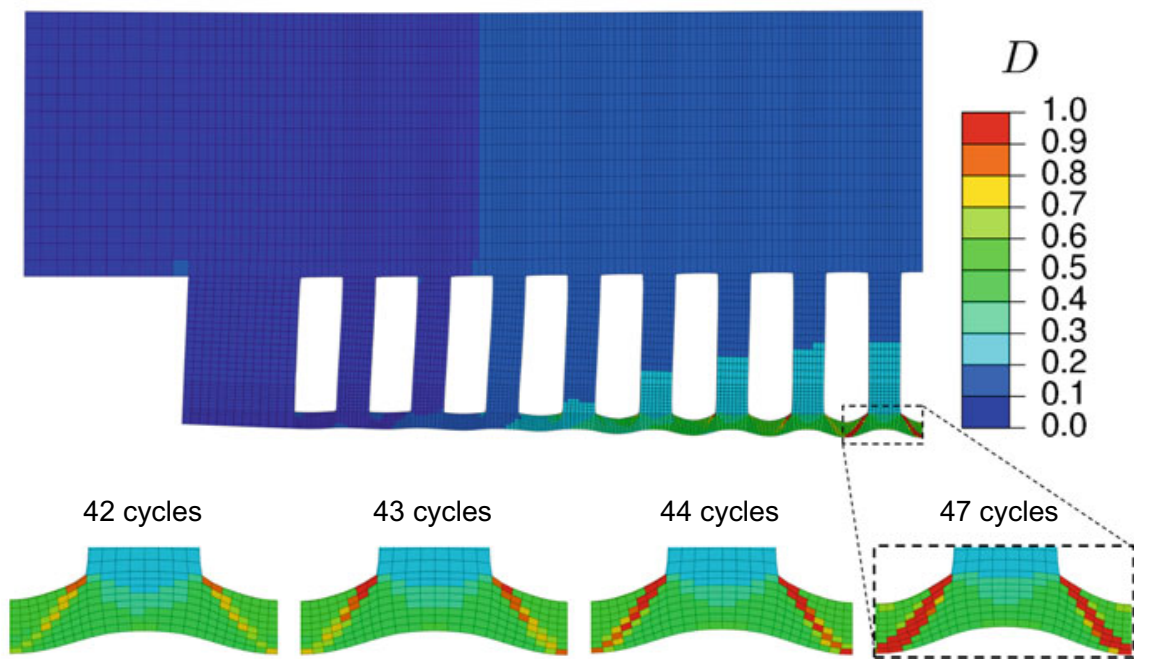

Fig. 7 Mechanical analysis results-(top) damage distribution after 47 cycles; (bottom) zoom into region of interest and damage evolution within last cycles

diagonally through the wall (cycle 43). Afterwards, damage starts to proceed also from the opposite hot gas side center (cycle 44) and merges eventually into a diagonal macroscopic crack (cycle 47). Additionally, Fig. 8 gives the reader an impression of the accumulation of damage during the simulation of one and 47 cycles within three different elements ${ }^{2}$ (i.e. cooling side edge, cooling side center, as well as hot gas side center). It is evident that damage increases most within the phase transitions of each cycle, since it is driven by the temperature gradient as well as the pressure difference between the coolant and the hot gas. This is why the maximum increase in damage takes place between the second hot run and the post cooling. During this transition phase, the greatest temperature and pressure differences occur. Furthermore, at this point the material properties are also the weakest here. Interestingly, when looking further at the development of damage over several cycles within the three considered elements (Fig. 8), it can be observed that the increase in damage is initially almost linear and then becomes highly non-linear. Particularly at the hot gas side center (red curve), an enormous increase in damage is observed after approx. 40 cycles, which leads to the crack spreading from both sides as already mentioned above.

In order to validate the numerical results from the quasi-static mechanical analysis also quantitatively, a comparison to experimental observations is made in Fig. 9. The cut view at the crack tip of the fatigue experiment after 48 cycles has been taken from the test campaign conducted by Hötte et al. [11]. After the 48th cycle, the breakthrough of the cooling channel (macroscopic failure) was first observed in the experiment. The maximum deformation of the specimen, which consists of bulging

\footnotetext{
${ }^{2}$ Note that since a single Gauss point FE formulation is utilized, the damage value in one element corresponds to its material point value (Quilt-type contours within Abaqus).
} 

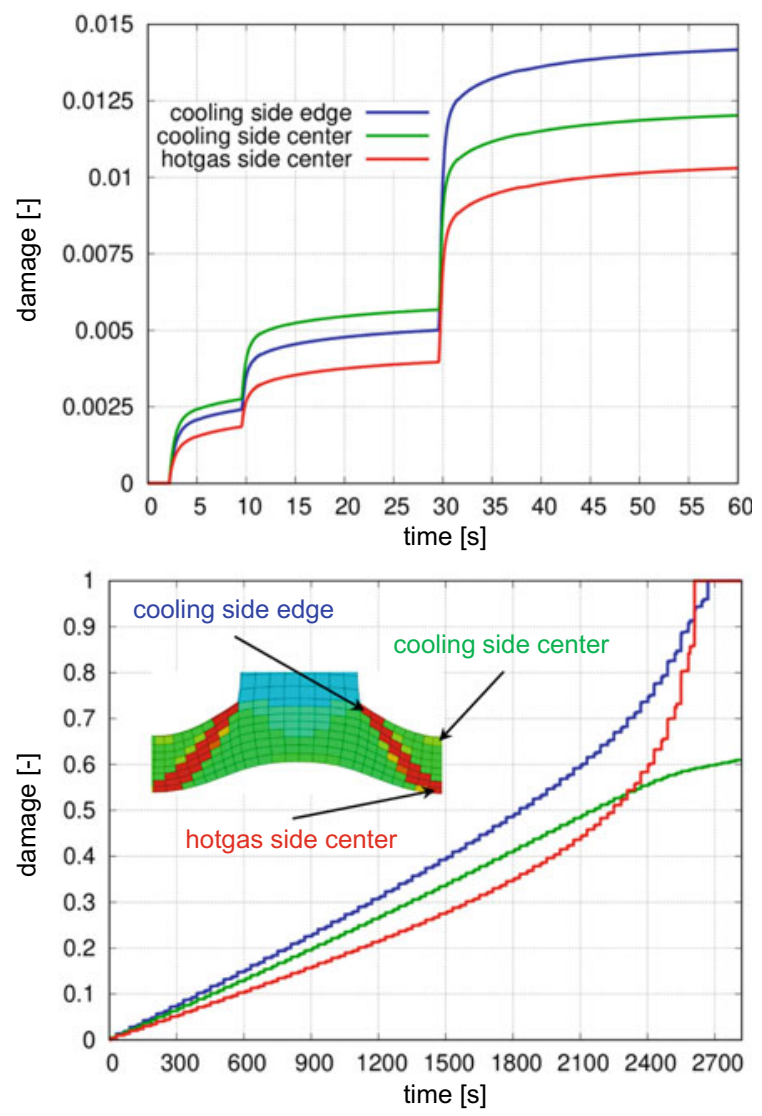

Fig. 8 Mechanical analysis results-(top) damage evolution during one cycle at three different positions; (bottom) damage evolution during 47 cycles at three different positions

and thinning of the hot gas wall, takes place at the cooling channel next to the symmetry plane. Whereas the bulging of the hot gas wall is accurately represented by the simulation, the thinning process can be interpreted in the following way. Since the damage variable reaches the value of $D=1$ within the diagonal process zone between the cooling side edge and the hot gas center, the elements above this region can no longer contribute to the load-bearing capacity of the specimen. Therefore, the elements above this process zone could also be thought of as being removed, which can ultimately be interpreted as thinning of the specimen. Since the deformed shape of the cooling channel wall after failure resembles the shape of a doghouse, the failure mode is frequently called doghouse effect in literature (see e.g. Riccius et al. [20]). In total, it can be stated that the doghouse effect can be well represented by the shown thermomechanical analysis scheme utilizing a viscoplastic damage model. 


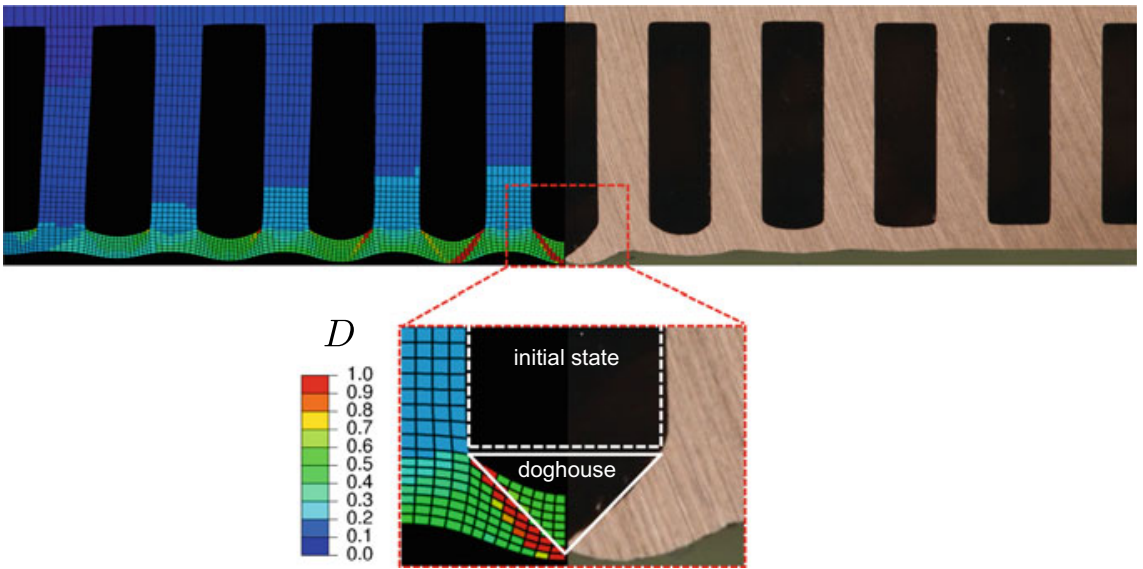

Fig. 9 Comparison with experimental observations-(left) deformed geometry and damage contour after 47 cycles obtained from simulation; (right) cut view of the fatigue experiment after 48 cycles showing a macroscopic crack in the center cooling channel [11]

\section{Conclusion}

In this work, a conjugate heat transfer model as well as the lifetime prediction of a rocket combustion chamber were addressed.

Concerning the former topic, a pseudo-transient conjugate heat transfer model of the fatigue experiment was developed. This model consists of the rectangular fatigue specimen, nine coolant flows and the hot gas flow. The sensitivity of the turbulent Prandtl number in the hot gas domain on the specimen's temperature was shown. The model showed a good agreement with the temperature distribution and transients of the experiment. Therefore, the model was able to predict the transient temperature fields of the structure without any Nusselt number correlation or experimental data fitting. This allowed to use the conjugate heat transfer model as a base for sequentially coupled lifetime predictions in different configurations, e.g. for cooling channel design optimization.

Regarding the second part of this contribution, a sequentially coupled thermomechanical analysis scheme for the lifetime prediction of the fatigue specimen was presented. First, a transient thermal analysis was carried out in order to obtain the temperature field within the specimen in every time step. Comparisons of the numerical results with thermocouple measurements close to the symmetry plane were in good agreement. Afterwards, the computed temperature served as input for a series of quasi-static mechanical analyses, in which a previously developed viscoplastic damage model was utilized. During the numerical analysis of the deformation process, it was found that the damage initially spreads diagonally from the cooling channel corner to the hot gas wall and eventually merges into a macroscopic failure zone. The comparison with the experiment showed that the number of cycles until failure, 
the position of maximum deformation and degradation, as well as the final failure mode (doghouse effect) were accurately predicted by the simulation.

Future work should focus, for example, on the extension of the material model to damage anisotropy. A corresponding framework was recently published by Fassin et al. [8], who introduced a damage tensor of second-order. The incorporation of tension-compression asymmetry, as presented by Fassin et al. [7], might be of interest as well. Furthermore, it would be worthwhile to examine the influence of potential finite element mesh dependencies, which might occur when using conventional, 'local' continuum damage models. A suitable approach for this was presented by Brepols et al. [5] for small strains and has only recently been extended to finite deformations by Brepols et al. [6]. The authors used a gradient-extension in order to obtain a non-local version of the model. Finally, the influence of specific finite element technologies based on reduced integration with hourglass stabilization should be investigated. Preliminary work in this regard can be found, for instance, in Reese et al. [19], Barfusz et al. [2, 4].

Acknowledgements Financial support has been provided by the German Research Foundation (Deutsche Forschungsgemeinschaft-DFG) in the framework of the Sonderforschungsbereich Transregio 40 .

\section{References}

1. ANSYS Fluent Theory Guide, 16 edn. Ansys Inc., Canonsburg (2017)

2. Barfusz, O., Brepols, T., Frischkorn, J., Reese, S.: Towards the incorporation of damage into solid-shells based on reduced integration. PAMM 19(1), e201900055 (2019)

3. Barfusz, O., Hötte, F., Reese, S., Haupt, M.C.: Lifetime analysis of a virtual thrust chamber demonstrator and 11-domain conjugate heat transfer simulation of a rocket combustion chamber. SFB TRR40 Annual Report 2019 (2019)

4. Barfusz, O., Smeenk, R., Reese, S.: Solid-shells based on reduced integration - geometrically non-linear analysis of layered structures. In: Proceedings of the 6th European Conference of Computational Mechanics (2018)

5. Brepols, T., Wulfinghoff, S., Reese, S.: Gradient-extended two-surface damage-plasticity: micromorphic formulation and numerical aspects. Int. J. Plast. 97, 64-106 (2017)

6. Brepols, T., Wulfinghoff, S., Reese, S.: A gradient-extended two-surface damage-plasticity model for large deformations. Int. J. Plast. accepted. Accessed 29 Nov 2019

7. Fassin, M., Eggersmann, R., Wulfinghoff, S., Reese, S.: Efficient algorithmic incorporation of tension compression asymmetry into an anisotropic damage model. Comput. Methods Appl. Mech. Eng. 354, 932-962 (2019)

8. Fassin, M., Eggersmann, R., Wulfinghoff, S., Reese, S.: Gradient-extended anisotropic brittle damage modeling using a second order damage tensor-theory, implementation and numerical examples. Int. J. Solids Struct. 167, 93-126 (2019)

9. Fassin, M., Hötte, F., Barfusz, O., Reese, S., Haupt, M.C.: Lifetime influence of thermal barrier coatings and thermal analysis of the fatigue experiment. SFB TRR40 Annual Report 2016, 8:227-237 (2016)

10. Fassin, M., Kowollik, D., Wulfinghoff, S., Reese, S., Haupt, M.C.: Design studies of rocket engine cooling structures for fatigue experiments. Arch. Appl. Mech. 86(12), 2063-2093 (2016) 
11. Hötte, F., Günther, O., Rohdenburg, M., Haupt, M.C., Scholz, P.: Roughness and crack investigations of rocket combustion chambers and pressure loss measurements in a high aspect ratio cooling duct. SFB TRR40 Annual Report 2019 (2019)

12. Hötte, F., Haupt, M.C.: Transient 3d conjugate heat transfer simulation of a rectangular goxgch4 rocket combustion chamber and validation. Aerosp. Sci. Technol. vol. 105 (2020)

13. Hötte, F., Lungu, P., von Sethe, C., Fiedler, T., Haupt, M.C., Haidn, O.: Experimental investigations of thermo-mechanical fluid-structure interaction in rocket combustion chambers. J. Propul. Power 35(5), 906-916 (2019)

14. Hötte, F., von Sethe, C., Fiedler, T., Haupt, M.C., Haidn, O.J., Rohdenburg, M.: Experimental lifetime study of regeneratively cooled rocket chamber walls. Int. J. Fatigue 138 (2020). https:// doi.org/10.1016/j.ijfatigue.2020.105649

15. Jones, W.P., Launder, B.E.: The prediction of laminarization with a two-equation model of turbulence. Int. J. Heat Mass Trans. 15(2), 301-314 (1972)

16. Kowollik, D., Tini, V., Reese, S., Haupt, M.: 3d fluid-structure interaction analysis of a typical liquid rocket engine cycle based on a novel viscoplastic damage model. Int. J. Numer. Methods Eng. 94, 1165-1190 (2013)

17. Menter, F.R., Garbaruk, A.V., Egorov, Y.: Explicit algebraic reynolds stress models for anisotropic wall-bounded flows. Prog. Flight Phys. 3, 89-104 (2012)

18. Niesner, R.: Gekoppelte Simulation thermisch-mechanischer Fluid-Struktur-Interaktionen für Hyperschall-Anwendungen. Shaker (2009)

19. Reese, S., Barfusz, O., Schwarze, M., Simon, J.-W.: Solid-shell formulations based on reduced integration - investigations of anisotropic material behaviour, large deformation problems and stability. In: Shell Structures: Theory and Applications, vol. 4, pp. 31-39. CRC Press (2017)

20. Riccius, J., Haidn, O., Zamataev, E.: Influence of time dependent effects on the estimated life time of liquid rocket combustion chamber walls. In: 40th Joint Propulsion Conference and Exhibit. Fort Lauderdale, Florida (2004)

21. Slavinskaya, N., Haidn, O.: Reduced chemical model for high pressure methane combustion with pah formation. In: 46th AIAA Aerospace Sciences Meeting and Exhibit (2008)

Open Access This chapter is licensed under the terms of the Creative Commons Attribution 4.0 International License (http://creativecommons.org/licenses/by/4.0/), which permits use, sharing, adaptation, distribution and reproduction in any medium or format, as long as you give appropriate credit to the original author(s) and the source, provide a link to the Creative Commons license and indicate if changes were made.

The images or other third party material in this chapter are included in the chapter's Creative Commons license, unless indicated otherwise in a credit line to the material. If material is not included in the chapter's Creative Commons license and your intended use is not permitted by statutory regulation or exceeds the permitted use, you will need to obtain permission directly from the copyright holder.

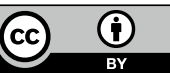

